Revista Arbitrada Interdisciplinaria KOINONIA

Año 2020. Vol V. №3. Especial: Administración

Hecho el depósito de Ley: FA2016000010

ISSN: 2542-3088

FUNDACIÓN KOINONIA (F.K). Santa Ana de Coro. Venezuela.

Jorge Patricio Cañar-Rivera; Johnny Hernan Urgiles-Vicuña; Juan Diego Ochoa-Crespo Mireya Magdalena Torres-Palacios

http://dx.doi.org/10.35381/r.k.v5i3.910

\title{
El mapa estratégico en las organizaciones públicas
}

The strategic map in public organizations

\author{
Jorge Patricio Cañar-Rivera \\ jorge.canar@psg.ucacue.edu.ec \\ Universidad Católica de Cuenca, Cuenca \\ Ecuador \\ https://orcid.org/0000-0002-0228-8058 \\ Johnny Hernán Urgiles-Vicuña \\ jhurgilesv@ucacue.edu.ec \\ Universidad Católica de Cuenca, Cuenca \\ Ecuador \\ https://orcid.org/0000-0001-8507-0400 \\ Juan Diego Ochoa-Crespo \\ jdochoac@ucacue.edu.ec \\ Universidad Católica de Cuenca, Cuenca \\ Ecuador \\ https://orcid.org/0000-0003-0781-2020 \\ Mireya Magdalena Torres-Palacios \\ mireya.torres@ucacue.edu.ec \\ Universidad Católica de Cuenca, Cuenca \\ Ecuador \\ https://orcid.org/0000-0002-7724-3313
}

Recibido: 20 de mayo de 2020

Revisado: 15 de junio de 2020

Aprobado: 30 de julio de 2020

Publicado: 15 de agosto de 2020 


\title{
RESUMEN
}

La investigación se basó en formular un sistema de control de la información con la finalidad de obtener un mapa estratégico y confiable del IESS en la provincia Cañar del Ecuador. Se trabajó metodológicamente desde la perspectiva descriptiva con diseño de campo no experimental, siendo la población de 91 empleados que conforman las unidades médicas de primer nivel de atención de salud en el área rural del IESS. Un 64\% de los encuestados afirman no recibir el apoyo necesario del planificador de la provincia, deduciéndose que existen falencias considerables en el área de planificación de la Dirección Provincial del IESS del Cañar, indicando la falta de planificadores calificados. En el ámbito de la seguridad social, es necesario anclar a los objetivos estratégicos la participación activa de sus afiliados, beneficiarios y empleados de todas sus unidades de negocio.

Descriptores: Seguridad social; administración pública; empresa pública; política de la salud. (Palabras Tomadas del Tesauro UNESCO).

\begin{abstract}
The research was based on formulating an information control system in order to obtain a strategic and reliable map of the IESS in the Cañar province of Ecuador. Methodological work was carried out from the descriptive perspective with a non-experimental field design, with the population of 91 employees that make up the first level medical units of health care in the rural area of the IESS. $64 \%$ of those surveyed affirm that they do not receive the necessary support from the provincial planner, deducing that there are considerable shortcomings in the planning area of the Provincial Directorate of the IESS del Cañar, indicating the lack of qualified planners. In the field of social security, it is necessary to anchor the active participation of its members, beneficiaries and employees of all its business units to the strategic objectives.
\end{abstract}

Descriptors: Social security; public administration; public enterprises; health policy. (Words Taken from the UNESCO Thesaurus). 


\section{INTRODUCCIÓN}

La Seguridad Social es un derecho consagrado en la Constitución de la República del Ecuador, la misma se basa en los principios de solidaridad, universalidad, equidad, eficiencia, subsidiaridad y suficiencia, el Instituto Ecuatoriano de Seguridad Social (IESS) protege a la población tanto urbana como rural contra las contingencias de enfermedad, maternidad, riesgos del trabajo, vejez, invalidez y muerte.

EI IESS tiene una amplia historia en el Ecuador, siendo creada la caja de pensiones el 8 de marzo de 1928 en el gobierno del doctor Isidro Ayora Cueva, sus objetivos fueron conceder a los empleados públicos, civiles y militares, los beneficios de Jubilación, Montepío Civil y Fondo Mortuorio, posteriormente en el año 1935 se crea el Instituto Nacional de Previsión, seguido en el año 1937 se crea la Caja del Seguro Social, incorporando en ese año el departamento médico con un desempeño administrativo y autónomo; en 1963 se fusionan las dos cajas citadas anteriormente y se crea la Caja Nacional del Seguro Social bajo la dirección del ex Instituto Nacional de Previsión, al siguiente año se incorpora el Seguro de riesgos del trabajo, el Seguro artesanal, el Seguro de profesionales, el Seguro de Trabajadores domésticos y por último en 1966, el Seguro del Clero Secular.

En el año 1970 se establece el Instituto Ecuatoriano de Seguridad Social, incorporan una década después, el Seguro Social Campesino para la población rural que se dedican netamente a labores de campo, el seguro obligatorio del trabajador agrícola y el seguro voluntario; de hecho, el IESS ofrece las siguientes prestaciones:

1. Seguro de Salud Individual y Familiar.

2. Seguro de Invalidez, Vejez y Muerte

3. Seguro de Riesgos del Trabajo.

4. Seguro Social Campesino.

5. Cesantía y Desempleo. (IESS, 2019) 
En este contexto una verdadera seguridad social deberá conceder una prestación íntegra de salud en todas las etapas de vida tanto de los ciudadanos ecuatorianos como los extranjeros residentes en territorio nacional del Ecuador, concediendo de manera igual prestaciones económicas a sus beneficiarios de acuerdo con su base de aportación personal como patronal, sean esas por enfermedad, incapacidad para laborar o por no percibir ingresos a causa del desempleo (Posso, 2005).

La seguridad Social en el Ecuador se centra en cubrir necesidades mínimas frente a ciertas contingencias como los son la enfermedad, riesgos del trabajo, desempleo, vejez y muerte, de esta manera otorgar dignidad a sus afiliados y beneficiarios, en especial en la etapa de la vida en la cual la persona ve disminuida su capacidad de generar ingresos por sí misma, ya sea por invalidez o por vejez; si bien es cierto el estado garantiza el derecho a la seguridad social, el IESS se financia de una manera autónoma con las aportaciones personales de sus afiliados como los aportes patronales de los empleadores (Morales, 2013).

De acuerdo con el boletín estadístico N$^{\circ} 23$ publicados en la página web del IESS, esa entidad cuenta con un total de 3'250.758 de afiliados al seguro general obligatorio, tanto del sector público, privado e incluso voluntarios residentes en el Ecuador como residentes en el extranjero, 1'140.484 afiliados y beneficiarios del Seguro Social Campesino, 542.439 pensionistas del seguro de invalidez, vejez y muerte, riesgos del trabajo y del seguro campesino, por último existen 1'032'370 beneficiarios no afiliados inscritos en el IESS.

En el caso de la provincia del Cañar, el número de afiliados asciende a la suma de 35.000 y alrededor de 20.000 afiliados y beneficiarios del seguro social campesino, así como existe aproximadamente 6.000 pensionistas, quienes demandan todos los servicios que el IESS ofrece. (IESS, 2019) 


\section{Jorge Patricio Cañar-Rivera; Johnny Hernan Urgiles-Vicuña; Juan Diego Ochoa-Crespo Mireya Magdalena Torres-Palacios}

\section{Tabla 1.}

Número de afiliados en la provincia del Cañar.

\begin{tabular}{lr} 
Cantón & No Afiliados 2019 \\
\hline Azogues & 15,128 \\
Biblián & 2,055 \\
\hline Cañar & 6,081 \\
\hline Deleg & 360 \\
\hline El Tambo & 803 \\
\hline La Troncal & 10,975 \\
\hline Suscal & 282 \\
\hline Cañar & 35,684 \\
\hline
\end{tabular}

Fuente: IESS 2019.

Para cubrir la demanda de servicios que sus afiliados, pensionistas y beneficiarios requieren, el IESS en la Provincial del Cañar cuenta con tres unidades médicas de primer nivel de atención distribuidas en los cantones de Azogues, Cañar y la Troncal, así mismo existe un hospital de segundo nivel de atención, Ilamado Centro Clínico Quirúrgico Hospital del día Azogues, siendo este, uno de los principales soportes del Hospital de Especialidades José Carrasco Arteaga, por otro lado, para abastecer la demanda de los servicios de salud en el sector rural el IESS mantiene 28 dispensarios del seguro social campesino operativo en todos los cantones de la provincia que brindan servicios de medicina general y odontología, por último existe una unidad administrativa en la ciudad de Azogues y una ventanilla de atención universal en el cantón La Troncal, que ofrecen los servicios de pensiones, riesgos del trabajo, fondos de terceros, seguro de desempleo, afiliación, entre otros (Mendieta-Ortega, et al., 2020). 
La Dirección Provincial del IESS del Cañar desempeña un papel importante en la gestión de todas las unidades de negocio del IESS en la provincia, y de acuerdo con lo que estipuló la resolución de Consejo Directivo CD 535 es el ente coordinador y jerárquicamente superior en el territorio provincial, dicha dependencia se encuentra ubicada en la ciudad de Azogues en las calles Veintimilla y Ayacucho, para el efecto entre otras unidades cuentan con la Unidad Provincial de Planificación, la misma que se encarga de consolidar resultados provinciales en el sistema de gestión por resultados, dichos resultados servirá a las Autoridades para tomar decisiones.

Una de las problemáticas en las dependencias administrativas y médicas del IESS en la provincia del Cañar e incluso a nivel nacional es la inexistencia de planificadores o personal capacitado en planificación estratégica, lo que ha generado que la información inscrita en el sistema de gestión por resultados del IESS de cierta manera no sea muy confiable, ya que se ha vuelto bastante complicado verificar la veracidad de la información que es suministrada por cada área operativa, quienes tratan de demostrar eficiencia absoluta en sus gestiones y no las falencias en ciertos procesos que requerirían ser analizados a más detalle por las autoridades de la institución, para que se planteen mejoras para el bienestar de sus afiliados, beneficiarios y la Institución como tal; la carencia de planificadores ha generado también que la información disponible no sea objeto de análisis de sus resultados y planteamientos para mejorar; razón por la cual se plantea formular un sistema de control de la información con la finalidad de obtener un mapa estratégico y confiable del IESS en la provincia Cañar del Ecuador.

\section{Referencial teórico}

\section{El mapa estratégico en las organizaciones}

La estrategia desempeña un papel importante en la existencia de todas las empresas cualquiera que sea su tipo de negocio, (kaplan \& Norton, 2004) refiere que la estrategia en una empresa se orienta a describir las formas en que se busca generar valor para sus propietarios y usuarios, los mismos que deberían estar alineados a sus activos 
Jorge Patricio Cañar-Rivera; Johnny Hernan Urgiles-Vicuña; Juan Diego Ochoa-Crespo Mireya Magdalena Torres-Palacios

intangibles, anteriormente cada organización planteaba sus estrategias desde diferentes puntos de vista, considerando aspectos como los financieros, productos o servicios, ventas, clientes, procesos internos, o talento humano, visto cada uno de ellos de manera independiente.

De igual manera (Tarjizán, 2018) expone que la estrategia en las organizaciones se basan en dos campos muy amplios cómo es la estrategia competitiva que busca identificar cuál es su ventaja competitiva, de qué manera sostenerla en un futuro y buscar los mecanismos para que la empresa tenga una mejor posición en el mercado y por otro lado la estrategia corporativa que busca analizar cuáles serían los negocios que las organizaciones deberían inclinar sus esfuerzos en miras de conseguir maximizar sus logros.

Es también relevante mencionar lo manifestado por (Porter, 2015) en cuanto a cómo una empresa deberá elaborar una estrategia competitiva, la cual deberá considerar como ésta lidiará en su industria, cuáles serán sus metas y que métodos empleará para conseguirlas, una de las maneras de cómo establecer dicha estrategia es analizar los cuatro factores que engloban a la organización, como son: las fortalezas, debilidades y los valores de los ejecutivos y empleados que definirán los factores internos de la empresa, de forma igual se analizarán los factores externos tales como las amenazas, oportunidades y las expectativas sociales (López-Zapata, et al., 2019).

Antes de estudiar a fondo el mapa estratégico en las organizaciones debemos entender el concepto de cuadro de mando integral, también conocido como balanced scorecard, (Rivero-Alonso \& Galarza-López, 2017), manifiestan que es la técnica de gestión que utilizan las empresas para plasmar su estrategia y convertirlos en objetivos claros y medibles y que estos tengan una relación estrecha, considerando que los actuaciones de los colaboradores de la empresa y sus recursos se encuentren estratégicamente relacionados, a fin de generar valor en la organización.

En ese mismo contexto (Kaplan \& Norton, 2014) el cuadro de mando integral resalta la necesidad de incluir a indicadores financieros y no financieros, de esta manera los 
Jorge Patricio Cañar-Rivera; Johnny Hernan Urgiles-Vicuña; Juan Diego Ochoa-Crespo Mireya Magdalena Torres-Palacios

empleados comprenderán como sus aportes afectarán las finanzas de su empresa, por otro lado los directivos analizarán los éxitos financieros a un tiempo largo, el cuadro de mando integral no solo es un resumen de los objetivos e indicadores, el mismo debe buscar convertir los objetivos y la estrategia en objetivos claros con indicadores perceptibles.

Los indicadores deberán estar conectados entre los que demuestren resultados pasados con los que incentiven esfuerzos futuros, en la actualidad las empresas están utilizando el cuadro de mando integral no solo como un sistema de medición, sino que lo utilizan como un sistema de gestión de su estrategia a largo plazo. Por consiguiente, se puede entender lo que implica el mapa estratégico, el mismo que a decir de (Martínez-Pedrós \& Milla-Gutierrez, 2012), manifiestan que el mapa estratégico describe de una manera clara y objetiva la estrategia de la empresa, en miras de establecer sus objetivos y metas y a más de aquello definir de qué manera se los va a conseguir, es decir deberá constar la estrategia planteada y la manera de gestionarla, demostrará también como la estrategia se enlaza con los activos intangibles con los procesos que generan valor (GonzálezCastillo, et al., 2017).

De igual manera, a partir de varios aspectos como los accionistas, clientes, productos, innovación, gestión del recurso humano y tecnologías de la información, el cuadro de mando integral se convierte en un sistema para formular y llevar a cabo la estrategia en una organización, por ende en el mapa estratégico se puede vincular todos los indicadores estratégicos de la empresa entre las cuatro perspectivas que plantea el cuadro de mando integral, no de manera independiente si no como una relación causa efecto entre los objetivos de todas las perspectivas (Lazo-Torres, et al., 2019).

En consecuencia, luego del modelo del cuadro de mando integral en el mapa estratégico se incluye la dinámica de la estrategia, la cual es descrita de una manera muy clara y coherente, de tal manera que los objetivos e indicadores puedan ser claramente gestionados, en resumen, el mapa estratégico enlaza el planteamiento de la estrategia con su ejecución (Kaplan \& Norton, 2014). 
De igual manera (Ortiz-Pérez, et al., 2014) manifiestan que el mapa estratégico representa una imagen de todos los aspectos que forman parte de la estrategia, es un mecanismo mediante el cual se puede apreciar como los recursos intangibles de una organzación se relaciona con otros recursos, sean estos tangibles o intangibles, en busca de generar valor tanto a los clientes como a los resultados financieros, buscando una forma clara de plantear la estrategia y los mecanismos de cómo desarrollarla con la finalidad de conseguir los objetivos planteados, en resumen es una unión entre la estrategia y la gestión de la estrategia (Erazo-Álvarez, et al., 2020).

\section{Aspectos que las organizaciones actuales consideran para formular los mapas estratégicos}

El mapa estratégico se establece a partir de la interacción de las cuatro perspectivas que se incluye en el cuadro de mando integral.

1. Perspectiva financiera. - implica a los objetivos netamente monetarios que tienen que ver con maximizar los ingresos contra la minimización de sus costos totales, se considera obtener la mejor rentabilidad posible en el corto plazo con proyección a obtener una mejor rentabilidad de sus capitalizaciones en el largo plazo.

2. Perspectiva del cliente. - satisfacer las necesidades del cliente es el factor más importante de la estrategia en la cual se plantea cuatro aspectos a considerar como son: mejor costo total, liderazgo de productos, soluciones completas a los clientes y sistema de bloqueo.

3. Perspectiva del proceso interno.- al contrario de las dos primeras perspectivas que se centran netamente en aspectos económicos como maximizar utilidades o ganar espacio de mercado a través de la captación de sus clientes, la perspectiva del proceso interno se concentra en identificar ciertos procesos claves que aporten directamente a incrementar su productividad enfatizando aspectos operacionales, clientes, de innovación y sociales, a los cuales se los considerará como temas estratégicos, en resumen se destaca que los resultados de los procesos 
Jorge Patricio Cañar-Rivera; Johnny Hernan Urgiles-Vicuña; Juan Diego Ochoa-Crespo Mireya Magdalena Torres-Palacios

operacionales se obtendrán en un corto plazo, seguido de los procesos con los clientes, mientras que los procesos de innovación y sociales pueden exigir mayor tiempo para la consecución de mejores resultados.

4. Perspectiva del aprendizaje.- la última perspectiva del balanced scorecard tienen que ver con los activos intangibles de la organización y están ligados con el capital humano, tecnologías de la información y el capital corporativo, los mismos que en la estrategia deberán ser evaluados guardando una relación directa entre cada uno de ellos, aunque si bien es cierto en la mayoría de casos las actividades del talento humano y tecnologías de la información no se encuentran relacionados con su estrategia, es de suma importancia que dichos procesos se encuentren alineados a la estrategia para mejorar la productividad de la organización (kaplan \& Norton, 2004).

Dentro de este marco es importante analizar también lo expuesto por (Herrera-Avendaño, et al., 2014) quienes afirman que el mapa estratégico se formulan a partir de los principios de equilibrar las fuerzas contradictorias, propuesta de valor diferenciado para el cliente, temas simultáneos y complementarios, el valor de los recursos intangibles.

\section{Las empresas y su generación de valor}

Como se ha analizado hasta el momento ninguna empresa deberá trasladar su mayor esfuerzo en cada uno de sus objetivos, esto dependerá de la estrategia que la organización se haya planteado, es decir si una empresa busca sobresalir en sus productos deberá enfocarse en procesos que tienen que ver con innovación, mientras que empresas que buscan minimizar sus costos trasladarán su mayor esfuerzo en los procesos de gestión de operaciones, insistiendo que cada uno de los objetivos estratégicos tendrán que estar alineados y unidos para conseguir aumentar su valor (Cordero-Naspud, et al., 2020).

Los tipos de estrategia que empleen las organizaciones son modos de formular la posición de la empresa dentro de la cadena de valor, de modo de obtener una significativa 
ganancia entre sus precios de venta y sus costos de producción, es necesario puntualizar si la oferta de una empresa no es única frente a la competencia, ésta mantendrá márgenes de ganancia bajos ya que los clientes absorben una buena parte del valor, en resumen una estrategia muy bien planteada en una unidad de negocio llevará a la empresa a ser competitiva y por ende captará gran parte del valor generado y luego de ello la organización podrá plasmar su estrategia en un cuadro de mapa estratégico y cuadro de mando integral (kaplan \& Norton, 2004).

\section{MÉTODO}

Se trabajó metodológicamente desde la perspectiva descriptiva con diseño de campo no experimental, siendo la población conformada por 91 empleados que conforman las unidades médicas de primer nivel de atención de salud en el área rural del IESS en la provincia del Cañar, previa autorización del señor Director Provincial del IESS Cañar. En lo que respecta a las técnicas, se utilizaron encuestas con la finalidad de levantar toda la información posible, para poder analizar las variables. Por tal motivo, el instrumento utilizado fue un cuestionario, estructurado con 12 preguntas de selección múltiple, siendo procesada la información recopilada mediante estadística descriptiva.

\section{RESULTADOS}

Una vez que se han aplicado los instrumentos de investigación se obtuvieron los resultados que a continuación se exponen:

El $41 \%$ de los empleados a quienes se les consultó, manifiestan estar en desacuerdo respecto si tienen conocimiento sobre los objetivos estratégicos del IESS, sumado a un $13 \%$ que están en total desacuerdo, siendo la mayoría de empleaos que no tienen conocimiento sobre los objetivos estratégicos de la Institución, lo cual revela una socialización baja del plan estratégico de la Institución.

De igual manera el $46 \%$ y el $11 \%$ de los encuestados manifiestan estar en desacuerdo y en total desacuerdo respectivamente, referente a que, si los objetivos de su unidad de 
negocio fueron debidamente socializados, frente a un minoritario $31 \%$ que dicen estar de acuerdo que dichos objetivos fueron socializados, lo cual sigue revelando las deficiencias respecto a la socialización del plan estratégico y de los objetivos de una unidad de negocio.
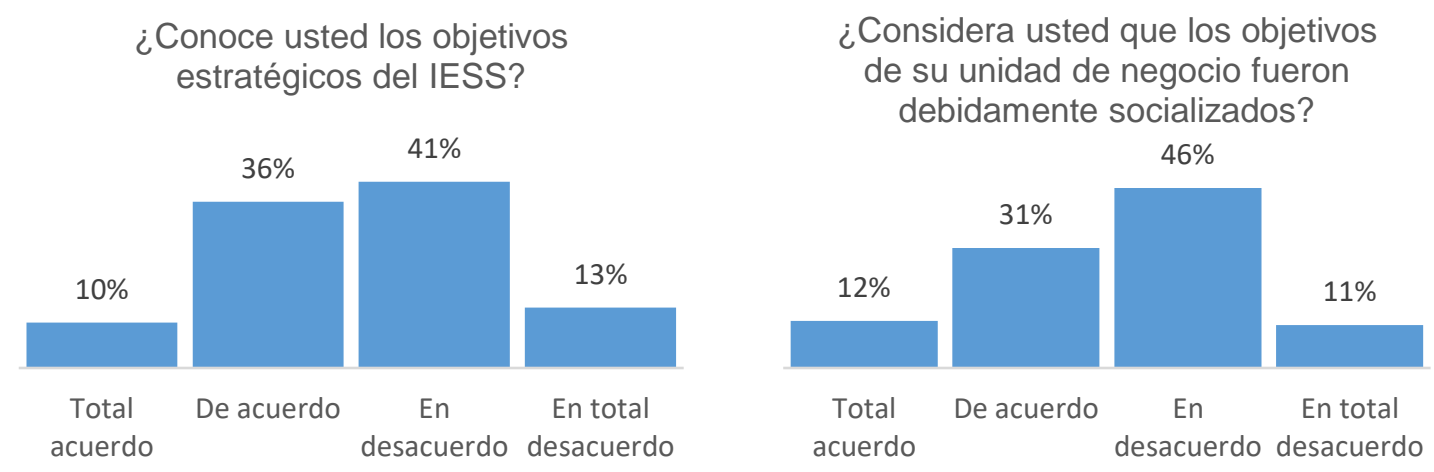

Figura 1. Objetivos estratégicos.

El $42 \%$ de los consultados indican estar de acuerdo que los objetivos de sus unidades deben ser conocidas por otras unidades de la Institución y un importante $22 \%$ afirman estar en total acuerdo con el tema.

El $47 \%$ de los encuestados señalan estar en desacuerdo que los objetivos planteados van acorde a la realidad de su unidad, frente a un 35\% que manifiestan estar de acuerdo y tan solo un $8 \%$ afirman estar en total acuerdo.

El $46 \%$ de los empleados consultados afirman estar en desacuerdo respecto a conocer cuáles son las estrategias de su unidad de negocio y el $11 \%$ señalan estar en total desacuerdo, lo que nos indica que una mayoría preocupante desconocen cuáles son las estrategias de su unidad de negocio en miras de conseguir los objetivos propuestos y apenas el $34 \%$ señalan estar de acuerdo en conocer dichas estrategias.

El $44 \%$ de los encuestados consideran estar en desacuerdo que las actividades por ellos realizadas estén enmarcadas de acuerdo con la estrategia de la Institución, sin embargo, 
existe un considerable $18 \%$ más un $30 \%$ que afirman estar en total acuerdo y de acuerdo respectivamente en que sus actividades están apegadas a la estrategia institucional.

¿Conoce usted cuales son las estrategias de su unidad de negocio para conseguir los objetivos?

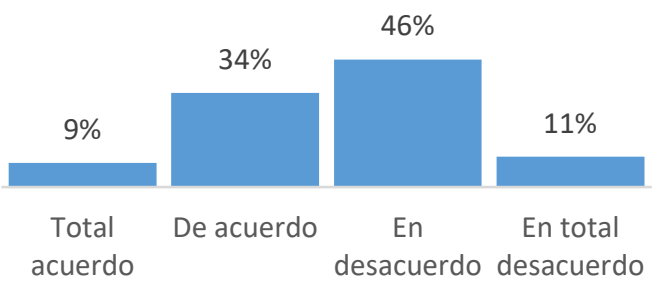

¿Considera usted que sus actividades van enmarcadas de acuerdo a la estrategia de la Institución?

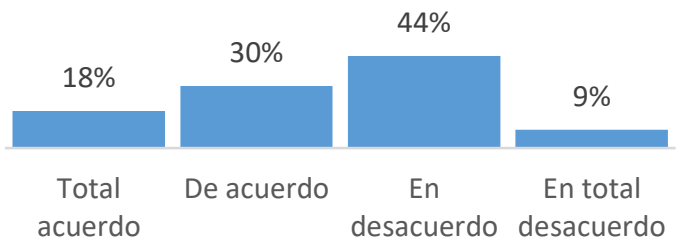

Figura 2. Estrategias de negocio.

Un 55\% de los empleados consultados exponen no tener los mecanismos suficientes para obtener información respecto a su gestión, lo que nos lleva a ver mecanismos para generar información y mantener un acceso fácil a la misma.

El $45 \%$ de los encuestados señalan estar en desacuerdo respecto a conocer el avance de consecución de sus objetivos y apenas un 33\% indican estar de acuerdo en conocerlos, lo que nos puede llevar a señalar una falencia en el seguimiento que se le da las metas planteadas.

Un importante $51 \%$ de los consultados afirman estar en desacuerdo con la retroalimentación recibida, respecto a los resultados obtenidos de su gestión y tan solo un $33 \%$ si están de acuerdo.

Un relevante $59 \%$ de los empleados indican que luego de terminado el periodo de gestión no reciben alguna información en relación con los resultados finales obtenidos en su unidad de negocio.

Por último, un 64\% de los encuestados afirman no recibir el apoyo necesario del planificador de la provincia, razón por la cual, se puede deducir que existen falencias considerables en el área de planificación de la Dirección Provincial del IESS del Cañar, 
Jorge Patricio Cañar-Rivera; Johnny Hernan Urgiles-Vicuña; Juan Diego Ochoa-Crespo Mireya Magdalena Torres-Palacios

lo que corrobora lo que se señaló al principio del presente estudio, la falta de planificadores calificados que mantiene la institución.

\section{¿Considera usted que recibe \\ retroalimentación respecto a los resultados obtenidos?}

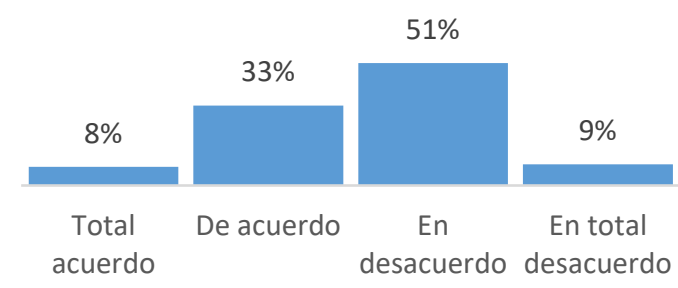

¿Conoce usted el avance de consecución de objetivos de su Unidad?

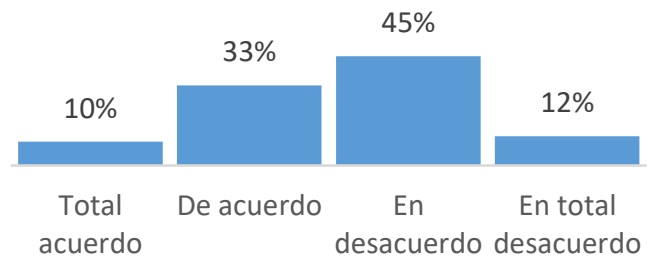

Figura 3. Objetivos de la unidad administrativa.

Tomando como punto de partida los resultados del diagnóstico realizado anteriormente se estable la propuesta del mapa estratégico para las unidades médicas de primer nivel de atención de salud en el área rural del IESS en la provincia del Cañar, teniendo en cuenta que las citadas unidades médicas forman parte de la Unidad Provincial del Seguro Social Campesino y esa a su vez es coordinada por la Dirección Provincial del Cañar, las cuales se rigen de acuerdo con el reglamento orgánico funcional del IESS. La misión y visión de dichas unidades médicas se plantean a partir de lo que establece la institución a nivel nacional. Basado en el desarrollo del mapa estratégico se diseña una guía gráfica para su ejecución: 


\section{Jorge Patricio Cañar-Rivera; Johnny Hernan Urgiles-Vicuña; Juan Diego Ochoa-Crespo Mireya Magdalena Torres-Palacios}

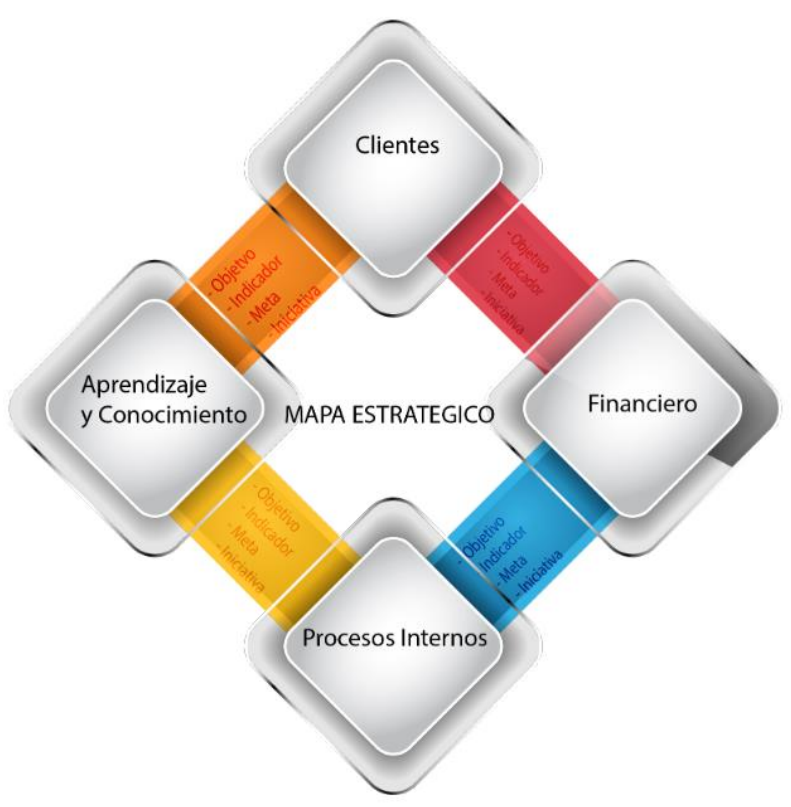

Figura 4. Guía gráfica del mapa estratégico.

\section{Misión}

Proteger a la población urbana y rural, con relación de dependencia laboral o sin ella, contra las contingencias de enfermedad, maternidad, riesgos del trabajo, discapacidad, cesantía, invalidez, vejez y muerte.

\section{Visión}

El plan estratégico sustentado en la Ley de Seguridad Social vigente convertirá a esta institución en una aseguradora moderna, técnica, con personal capacitado que atenderá con eficiencia, oportunidad y amabilidad a toda persona que solicite los servicios y prestaciones que ofrece. 
Jorge Patricio Cañar-Rivera; Johnny Hernan Urgiles-Vicuña; Juan Diego Ochoa-Crespo Mireya Magdalena Torres-Palacios

\section{Matriz FODA}

En base al diagnóstico realizado se estable las siguientes debilidades, fortalezas, amenazas y oportunidades, organizadas en las siguientes Matriz FODA.

\section{Tabla 2}

\section{Matriz FODA}

\section{FORTALEZAS}

F1- Cumplimiento de la normativa institucional.

F2- La institución tiene establecidos objetivos estratégicos.

F3- Seguro establecido en el mercado y altamente competitivo

La institución cuenta con

F4- infraestructura propia en el $93 \%$ de las unidades médicas del sector rural.

\section{DEBILIDADES}

D1- Ausencia de personal capacitado para la planificación estratégica.

D2- Ausencia de personal en la gestión documental.

D3- Déficit del personal de apoyo médico.

D4- Falta de confiabilidad de la información de la institución.

D5- Falta de control por parte de las autoridades de la institución.

D6- Falta de insumos y medicamentos en las unidades médicas.

Falta de conocimiento por parte de

D7- los trabajadores sobre los objetivos estratégicos del IESS.

Los objetivos establecidos no están

D8- acorde a la situación de cada unidad de negocio.

D9- No existe retroalimentación sobre los resultados de la institución.

Procesos centralizados que limitan la

D10- gestión de la institución a nivel provincial. 
Jorge Patricio Cañar-Rivera; Johnny Hernan Urgiles-Vicuña; Juan Diego Ochoa-Crespo Mireya Magdalena Torres-Palacios

\section{OPORTUNIDADES}

Diferentes instituciones locales dispuestas a establecer convenios para fortalecer la presencia del IESS.

Personal médico y de apoyo

O2- dispuestos a laborar en las unidades médicas del IESS.

O3- Creciente demanda de los servicios del seguro campesino. Instituciones públicas y privadas O4- dispuestas a contratar con el IESS.

\section{AMENAZAS}

Desconocimiento de la normativa del

A1- seguro campesino por parte de los asegurados.

Incumplimiento de la entrega de

A2- medicamentos por parte de los proveedores.

A3- Crisis sanitaria por el COVID-19.

A4- Crisis económica a nivel nacional.

A5-

Incumplimiento de las obligaciones económicas por parte del Gobierno.

A6Cambios constantes en el sistema de contratación pública.

Teniendo en cuenta las debilidades, fortalezas, oportunidades y amenazas identificadas se establece la siguiente matriz estratégica en post de potenciar las fortalezas y atenuar las debilidades de la institución y que aquello contrarreste las amenazas del entorno y se aproveche de mejor manera las oportunidades. A continuación, se establece la matriz estratégica. 
Revista Arbitrada Interdisciplinaria KOINONIA

Año 2020. Vol V. №3. Especial: Administración

Hecho el depósito de Ley: FA2016000010

ISSN: 2542-3088

FUNDACIÓN KOINONIA (F.K). Santa Ana de Coro. Venezuela.

Jorge Patricio Cañar-Rivera; Johnny Hernan Urgiles-Vicuña; Juan Diego Ochoa-Crespo Mireya Magdalena Torres-Palacios

Tabla 3.

Matriz estratégica.

AMENAZAS

OPORTUNIDADES

\begin{tabular}{ccl}
\hline Externas & A1- & $\begin{array}{l}\text { Desconocimiento de la } \\
\text { normativa del seguro }\end{array}$ \\
& campesino por parte de \\
& los asegurados
\end{tabular}

A2- Incumplimiento de la O2- Personal médico y de apoyo dispuestos entrega de medicamentos a laborar en las unidades médicas del por parte de los IESS

proveedores

A3- Crisis sanitaria por el O3- Creciente demanda de los servicios del COVID-19 seguro campesino

Internas A4- Crisis económica a nivel O4- Instituciones públicas y privadas nacional dispuestas a contratar con el IESS

A5- Incumplimiento de las obligaciones económicas por parte del Gobierno

A6- Cambios constantes en el sistema de contratación pública

\section{DEBILIDADES}

\begin{tabular}{lllll}
\hline D1- $\begin{array}{l}\text { Ausencia de personal } \\
\text { capacitado }\end{array}$ para la & D1 & Implementar un plan & D1O3 & $\begin{array}{l}\text { Contratar instituciones } \\
\text { para capacitar en }\end{array}$ \\
planificación estratégica. & & $\begin{array}{l}\text { de capacitación } \\
\text { considerando la } \\
\text { austeridad nacional }\end{array}$ & $\begin{array}{l}\text { planificación estratégica } \\
\text { al personal administrativo } \\
\text { de la institución }\end{array}$
\end{tabular}

D2- Ausencia de personal en D2A4 Implementar un plan la gestión documental

D3- Déficit del personal de D3A3 apoyo médico de reclutamiento para la institución

Identificar las brechas de personal médico y de apoyo en la institución
D3O2 Implementar un plan de reclutamiento de personal calificado para la institución 
Revista Arbitrada Interdisciplinaria KOINONIA

Año 2020. Vol V. №3. Especial: Administración

Hecho el depósito de Ley: FA2016000010

ISSN: 2542-3088

FUNDACIÓN KOINONIA (F.K). Santa Ana de Coro. Venezuela.

Jorge Patricio Cañar-Rivera; Johnny Hernan Urgiles-Vicuña; Juan Diego Ochoa-Crespo

Mireya Magdalena Torres-Palacios

D4- Falta de confiabilidad de la información de la institución

D5- Falta de control por parte de las autoridades de la institución.

D6- Falta de insumos y medicamentos en las unidades médicas

D7- Falta de conocimiento por parte de los trabajadores sobre los objetivos estratégicos del IESS

D8- Los objetivos establecidos no están acorde a la situación de cada unidad de negocio

$\begin{array}{ll}\text { D9- } & \text { No existe } \\ \text { retroalimentación } & \begin{array}{r}\text { sobre } \\ \text { los resultados de la } \\ \text { institución }\end{array}\end{array}$

D10- Procesos centralizados que limitan la gestión de la institución a nivel provincial
D4A4 Establecer un sistema de gestión de la información

D5A5 Implementar un programa de Control Interno basado en el coso III

D6A2 Establecer un programa de retroalimentación sobre los resultados donde se incluya al sector afiliado

D7A4 Establecer un plan de socialización de los objetivos de la institución

D8A5

Realizar un

diagnóstico

situacional de la

institución

\section{D9A1}

Establecer

un

programa de retroalimentación sobre los resultados donde se incluya al sector afiliado

D10A6

Priorizar los

procesos

desconcentrados

con una correcta aplicación de la Ley

D6O4 Diversificar
proveedores de los
institución

D801 Establecer objetivos estratégicos teniendo en cuenta el diagnóstico situacional de la institución con la participación de entidades del medio

FORTALEZAS

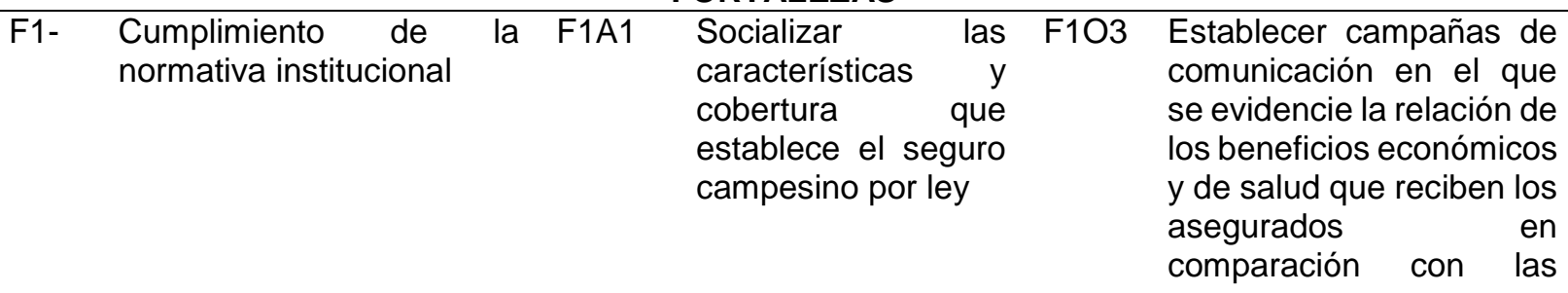


Revista Arbitrada Interdisciplinaria KOINONIA

Año 2020. Vol V. №3. Especial: Administración

Hecho el depósito de Ley: FA2016000010

ISSN: 2542-3088

FUNDACIÓN KOINONIA (F.K). Santa Ana de Coro. Venezuela.

Jorge Patricio Cañar-Rivera; Johnny Hernan Urgiles-Vicuña; Juan Diego Ochoa-Crespo

Mireya Magdalena Torres-Palacios

\begin{tabular}{|c|c|c|c|c|c|}
\hline & & & & & $\begin{array}{ll}\text { bajas aportaciones de } \\
\text { estos }\end{array}$ \\
\hline F2- & $\begin{array}{l}\text { La institución r tiene } \\
\text { establecidos objetivos } \\
\text { estratégicos. }\end{array}$ & F2A4 & $\begin{array}{l}\text { Mantener un control } \\
\text { sobre el nivel de } \\
\text { ejecución de los } \\
\text { objetivos } \\
\text { establecidos. }\end{array}$ & $\mathrm{F} 2 \mathrm{O} 2$ & $\begin{array}{l}\text { Establecer objetivos } \\
\text { estratégicos enfocados en } \\
\text { el desarrollo de los RRHH } \\
\text { de la institución. }\end{array}$ \\
\hline F3- & $\begin{array}{l}\text { Seguro establecido en el } \\
\text { mercado y altamente } \\
\text { competitivo }\end{array}$ & F3A1 & $\begin{array}{lr}\text { Establecer } & \\
\text { campañas } & \text { de } \\
\text { comunicación para } \\
\text { enfatizar r las } \\
\text { ventajas del seguro } \\
\text { campesino sobre } \\
\text { otros seguros. }\end{array}$ & F3O1 & $\begin{array}{l}\text { Establecer campañas de } \\
\text { comunicación para } \\
\text { enfatizar las ventajas del } \\
\text { seguro campesino sobre } \\
\text { otros seguros. }\end{array}$ \\
\hline F4- & $\begin{array}{l}\text { La institución cuenta con } \\
\text { infraestructura propia en el } \\
93 \% \text { de las unidades } \\
\text { médicas del sector rural. }\end{array}$ & F4A3 & $\begin{array}{lr}\text { Emplear la liquidez } \\
\text { recaudada } & \text { en } \\
\text { mejorar } & \text { la } \\
\text { infraestructura } & \\
\text { hospitalaria. } & \end{array}$ & & \\
\hline
\end{tabular}

Como punto de partida de las estrategias anteriormente definidas se estable un mapa estratégico, esta herramienta permite analizar la evolución de la organización, con lo cual se pueden tomar decisiones rápidas y certeras en caso de desviaciones, adaptar las estrategias empleadas o aplicar nuevas estrategias, siempre con la finalidad de hacer que la institución pueda cumplir con los objetivos propuestos. Se presentan los componentes del mapa estratégico 
Jorge Patricio Cañar-Rivera; Johnny Hernan Urgiles-Vicuña; Juan Diego Ochoa-Crespo Mireya Magdalena Torres-Palacios

Tabla 4.

Componentes del Cuadro de Mando Integral.

TÍTULO

Áreas o Perspectiva

Objetivos Estratégicos

Indicador

Frecuencia

Meta

Variaciones

\section{DEFINICIÓN}

Áreas de resultados clave de la empresa, 0 perspectivas.

En este campo de determinan los objetivos estratégicos, conforme las perspectivas.

Se establece el nombre del indicador.

Se establece la frecuencia con la que se realizará el control, puede ser diario, semanal, mensual, trimestral, anual.

Aquí se establece la meta que se quiere alcanzar.

El porcentaje de variación determina el porcentaje de la meta que se está alcanzando:

Óptimo (mayor que $66 \%$ )

$\bigcirc$ Tolerable (entre $33 \%$ y $65 \%$ )

Deficiente (entre $0 \%$ y $32 \%$ ) 
Revista Arbitrada Interdisciplinaria KOINONIA

Año 2020. Vol V. №3. Especial: Administración

Hecho el depósito de Ley: FA2016000010

ISSN: 2542-3088

FUNDACIÓN KOINONIA (F.K). Santa Ana de Coro. Venezuela.

\section{Jorge Patricio Cañar-Rivera; Johnny Hernan Urgiles-Vicuña; Juan Diego Ochoa-Crespo Mireya Magdalena Torres-Palacios}

\section{Seguidamente se desarrolla el mapa estratégico basado en las estrategias:}

\begin{tabular}{|c|c|c|c|c|c|c|c|c|}
\hline \multirow{2}{*}{ PERSPECTIVA } & \multirow{2}{*}{$\begin{array}{c}\text { OBJETIVOS } \\
\text { ESTRATÉGICOS }\end{array}$} & \multirow{2}{*}{ INICIATIVA } & \multirow{2}{*}{ INDICADOR } & \multirow{2}{*}{ FRECUENCIA } & \multirow{2}{*}{ META } & \multicolumn{3}{|c|}{ VARIACIONES } \\
\hline & & & & & & OPTIMO & TOLERABLE & DEFINICIENTE \\
\hline \multirow{3}{*}{ FINANCIERA } & \multirow{3}{*}{$\begin{array}{l}\text { Incrementar en } \\
\text { un } 1 \% \text { el } \\
\text { número de } \\
\text { afiliados del } \\
\text { seguro } \\
\text { campesino para } \\
\text { el } 2023 \text {. }\end{array}$} & $\begin{array}{l}\text { Determinar las } \\
\text { preferencias de los } \\
\text { campesinos de la } \\
\text { provincia Cañar. }\end{array}$ & $\mathrm{PC}=\frac{\text { No. de campesinos encuestados }}{\text { Total de la muestra }} * 100$ & Anual & $100 \%$ & $x>68 \%$ & $\frac{x 33 \%}{66 \%}$ & $x<32 \%$ \\
\hline & & $\begin{array}{l}\text { Identificar los } \\
\text { acuerdos } \\
\text { potenciales basado } \\
\text { en las preferencias } \\
\text { de los campesinos. }\end{array}$ & $\mathrm{AP}=\frac{\text { No. de despachos cumplidos }}{\text { Total de despachos requeridos }} * 100$ & Anual & $100 \%$ & $x>66 \%$ & $\frac{x \geq 33 \%}{66 \%}$ & $x<32 \%$ \\
\hline & & $\begin{array}{l}\text { Implementar } \\
\text { campañas de } \\
\text { comunicación en el } \\
\text { que se enfatiza las } \\
\text { ventajas del seguro } \\
\text { campesino sobre } \\
\text { otros seguros. } \\
\end{array}$ & $\mathrm{CC}=\frac{\text { Estartegias de comunicación }}{\text { Total de estrategias }} * 100$ & Anual & $100 \%$ & $x>66 \%$ & $\frac{x \geq 33 \% 6-}{66 \%}$ & $x<32 \%$ \\
\hline \multirow{7}{*}{ CLIENTE } & \multirow{3}{*}{$\begin{array}{l}\text { Mejorar en un } \\
10 \% \text { la } \\
\text { satisfacción del } \\
\text { afiliado del } \\
\text { senurn }\end{array}$} & $\begin{array}{l}\text { Establecer un } \\
\text { programa de } \\
\text { retroalimentación } \\
\text { sobre los resultados } \\
\text { donde se incluya al } \\
\text { sector afiliado. }\end{array}$ & $\mathrm{CR}=\frac{\text { Informes o que jas procesadas }}{\text { Total de Informes o que jas }} * 100$ & Trimestral & $100 \%$ & $x>68 \%$ & $\frac{x 33 \%-}{66 \%}$ & $x<32 \%$ \\
\hline & & $\begin{array}{l}\text { Plantear programa } \\
\text { de meinras ron la }\end{array}$ & & & & & & \\
\hline & & $\begin{array}{l}\text { Establecer } \\
\text { campañas de } \\
\text { comunicación en el } \\
\text { que se evidencie la } \\
\text { relación de los } \\
\text { beneficios } \\
\text { económicos y de } \\
\text { salud que reciben } \\
\text { los asegurados en } \\
\text { comparación con las } \\
\text { bajas aportaciones } \\
\text { de estos. }\end{array}$ & $\mathrm{CC}=\frac{\text { Estartegias de comunicación }}{\text { Total de estrategias }} * 100$ & Anual & $100 \%$ & $x>66 \%$ & $\frac{x \geq 33 \%}{66 \%}$ & $x<32 \%$ \\
\hline & \multirow{4}{*}{$\begin{array}{l}\text { Lograr que el } \\
80 \% \text { de afiliados } \\
\text { al seguro } \\
\text { campesino } \\
\text { comprendan la } \\
\text { nomativa del } \\
\text { seguro para el } \\
2025 \text {. }\end{array}$} & $\begin{array}{l}\text { Identificar los } \\
\text { locales en los que } \\
\text { se pudiera impartir } \\
\text { los talleres. }\end{array}$ & $\mathrm{LA}=\frac{\text { Locales inspeccionados }}{\text { Total de posibles locales }} * 100$ & Semestral & $100 \%$ & $x>66 \%$ & $\frac{x \geq 33 \%}{66 \%}$ & $x<32 \%$ \\
\hline & & $\begin{array}{l}\text { Determinar el } \\
\text { presupuesto de } \\
\text { gasto requerido para } \\
\text { impartir los talleres. } \\
\end{array}$ & $\mathrm{AP}=\frac{\text { No. de despachos cumplidos }}{\text { Total de despachos requeridos }} * 100$ & Semestral & $100 \%$ & $x>66 \%$ & $\frac{x \geq 33 \%}{66 \%}$ & $x<32 \%$ \\
\hline & & $\begin{array}{l}\text { Incluir los gastos en } \\
\text { el presupuesto de la } \\
\text { institución. }\end{array}$ & $\mathrm{PG}=\frac{\text { Monto incluido en el presupuesto }}{\text { Total de Gastos }} * 100$ & 0 Anual & $100 \%$ & $x>66 \%$ & $\frac{x \geq 33 \%}{66 \%}$ & $x<32 \%$ \\
\hline & & $\begin{array}{l}\text { Impartir los talleres } \\
\text { de comunicación } \\
\text { anual en que se } \\
\text { divulgue la } \\
\text { normativa del } \\
\text { seguro campesino. }\end{array}$ & $\mathrm{TC}=\frac{\text { Talleres inpartidos }}{\text { Total de talleres planificados }} * 100$ & Anual & $100 \%$ & $x>66 \%$ & $\frac{x \geq 33 \%}{66 \%}$ & $x<32 \%$ \\
\hline \multirow{3}{*}{$\begin{array}{l}\text { PROCESO } \\
\text { INTERNO }\end{array}$} & \multirow{3}{*}{$\begin{array}{l}\text { Establecer } \\
\text { objetivos } \\
\text { estratégicos } \\
\text { teniendo en } \\
\text { cuenta el } \\
\text { diagnóstico } \\
\text { situacional de la } \\
\text { institución con la } \\
\text { participación de } \\
\text { entidades del } \\
\text { medio para el } \\
2021 .\end{array}$} & $\begin{array}{l}\text { Realizar el análisis } \\
\text { situgcional de la } \\
\text { institución. } \\
\end{array}$ & $\mathrm{AP}=\frac{\text { No. de despachos cumplidos }}{\text { Total de despachos requeridos }} * 100$ & Anual & $100 \%$ & $x>66 \%$ & $\frac{x \geq 33 \%}{66 \%}$ & $x<32 \%$ \\
\hline & & $\begin{array}{l}\text { Establecer los } \\
\text { nuevos objetivos de } \\
\text { la institución de } \\
\text { acuerdo con el } \\
\text { diagnóstico } \\
\text { situacional. }\end{array}$ & $\mathrm{AP}=\frac{\text { No. de despachos cumplidos }}{\text { Total de despachos requeridos }} * 100$ & Anual & $100 \%$ & $x>66 \%$ & $\frac{x \geq 33 \%}{66 \%}$ & $x<32 \%$ \\
\hline & & $\begin{array}{l}\text { Establecer un plan } \\
\text { de socialización de } \\
\text { los objetivos de la }\end{array}$ & $\mathrm{PA}=\frac{\text { No. de actividades }}{\text { Total de actividades }} * 100$ & Anual & $100 \%$ & $x>66 \%$ & $\frac{x \geq 33 \%}{66 \%}$ & $x<32 \%$ \\
\hline
\end{tabular}


Revista Arbitrada Interdisciplinaria KOINONIA

Año 2020. Vol V. №3. Especial: Administración

Hecho el depósito de Ley: FA2016000010

ISSN: 2542-3088

FUNDACIÓN KOINONIA (F.K). Santa Ana de Coro. Venezuela.

\section{Jorge Patricio Cañar-Rivera; Johnny Hernan Urgiles-Vicuña; Juan Diego Ochoa-Crespo Mireya Magdalena Torres-Palacios}

\begin{tabular}{|c|c|c|c|c|c|c|c|c|}
\hline & & \multicolumn{5}{|l|}{ institución. } & \multirow[b]{2}{*}{$\frac{x \geq 33 \%}{66 \%}$} & \multirow[b]{2}{*}{$x<32 \%$} \\
\hline & & $\begin{array}{l}\text { Diseñar e } \\
\text { implementar un } \\
\text { Cuadro de Mando } \\
\text { Integral }\end{array}$ & $\mathrm{I}=\frac{\text { No. de indicadores inplementados }}{\text { Total de indicadores }} * 100$ & Anual & $100 \%$ & $x>66 \%$ & & \\
\hline & \multirow{2}{*}{$\begin{array}{l}\text { Cubrir el } 100 \% \\
\text { de las } \\
\text { necesidades de } \\
\text { recursos } \\
\text { humanos para el } \\
2021 .\end{array}$} & $\begin{array}{l}\text { Identificar las } \\
\text { brechas del } \\
\text { personal médico y } \\
\text { de apoyo en la } \\
\text { institución. } \\
\end{array}$ & $\mathrm{AP}=\frac{\text { No. de despachos cumplidos }}{\text { Total de despachos requeridos }} * 100$ & Anusl & $100 \%$ & $x>66 \%$ & $\frac{x \geq 33 \%}{68 \%}$ & $x<32 \%$ \\
\hline & & $\begin{array}{l}\text { Implementar un plan } \\
\text { de reclutamiento } \\
\text { para la institución. }\end{array}$ & $\mathrm{PA}=\frac{\text { No. de actividades }}{\text { Total de actividades }} * 100$ & Anual & $100 \%$ & $x>66 \%$ & $\frac{x \geq 33 \%}{66 \%}$ & $x<32 \%$ \\
\hline & \multirow{4}{*}{$\begin{array}{l}\text { Disminuir al } 0 \% \\
\text { el } \\
\text { desabastecimie } \\
\text { nto de insumos } \\
\text { y medicamentos } \\
\text { para el } 2022 .\end{array}$} & $\begin{array}{l}\text { Establecer un } \\
\text { sistema de alerta } \\
\text { temprana basada en } \\
\text { la gestión de } \\
\text { inventarios. }\end{array}$ & $\mathrm{AP}=\frac{\text { No. de despachos cumplidos }}{\text { Total de despachos requeridos }} * 100$ & Trimestral & $100 \%$ & $x>66 \%$ & $\frac{x \geq 33 \%}{66 \%}$ & $x<32 \%$ \\
\hline & & $\begin{array}{l}\text { Realizar procesos } \\
\text { de contratación } \\
\text { pública de manera } \\
\text { oportuna. } \\
\end{array}$ & $\mathrm{CP}=\frac{\text { Contrataciones oportunas }}{\text { Total de contrataciones }} * 100$ & Semestral & $100 \%$ & $x>66 \%$ & $\begin{array}{c}x \geq 33 \% \\
66 \%\end{array}$ & $x<32 \%$ \\
\hline & & \multirow{2}{*}{$\begin{array}{l}\text { Diversificar los } \\
\text { proveedores de la } \\
\text { institución. }\end{array}$} & $\mathrm{PA}=\frac{\text { No. de proveedores analizados }}{\text { Total de posibles proveedores }} * 100$ & Anual & $100 \%$ & $x>66 \%$ & $\frac{x \geq 33 \%}{66 \%}$ & $x<32 \%$ \\
\hline & & & $\mathrm{PR}=\frac{\text { No. de proveedores re gistrados }}{\text { Total de proveedores analizados }} * 100$ & Anual & $100 \%$ & $x>66 \%$ & $\begin{array}{c}x \geq 33 \% \\
66 \%\end{array}$ & $x<32 \%$ \\
\hline \multirow{6}{*}{$\begin{array}{l}\text { APRENDIZAJ } \\
\text { E }\end{array}$} & \multirow{4}{*}{$\begin{array}{l}\text { Establecer un } \\
\text { sistema de } \\
\text { gestión de la } \\
\text { información para } \\
\text { el } 2022 \text {. }\end{array}$} & $\begin{array}{l}\text { Diseñar un plan de } \\
\text { actualización de } \\
\text { recursos } \\
\text { tecnológicos. }\end{array}$ & $\mathrm{PA}=\frac{\text { No. de actividades }}{\text { Total de actividades }} * 100$ & Anual & $100 \%$ & $x>66 \%$ & $x \geq 33 \%$ & $x<32 \%$ \\
\hline & & $\begin{array}{l}\text { Levantamiento de } \\
\text { los procesos que se } \\
\text { llevan a cabo en }\end{array}$ & $\mathrm{P}=\frac{\text { Procesos existentes }}{\text { Procesos definidos }} * 100$ & Mensual & $100 \%$ & $x>66 \%$ & $\frac{x \geq 33 \%}{66 \%}$ & $x<32 \%$ \\
\hline & & cada tramite. & & & & & & \\
\hline & & $\begin{array}{l}\text { Informatización de } \\
\text { los trámites. }\end{array}$ & $\mathrm{T}=\frac{\text { Tramites existentes }}{\text { Tramites informatizados }} * 100$ & Mensual & $100 \%$ & $x>66 \%$ & $\frac{x \geq 33 \%}{66 \%}$ & $x<32 \%$ \\
\hline & \multirow{2}{*}{$\begin{array}{l}\text { Certificar para el } \\
2023 \text { que la } \\
\text { información } \\
\text { generada sea el } \\
100 \% \text { confiable. }\end{array}$} & $\begin{array}{l}\text { Implementar un plan } \\
\text { de capacitación } \\
\text { anual considerando } \\
\text { la austeridad } \\
\text { nacional. }\end{array}$ & $\mathrm{PA}=\frac{\text { No. de actividades }}{\text { Total de actividades }} * 100$ & Anual & $100 \%$ & $x>66 \%$ & $\frac{x \geq 33 \%}{66 \%}$ & $x<32 \%$ \\
\hline & & $\begin{array}{l}\text { Implementar un } \\
\text { sistema de control } \\
\text { interno basado en } \\
\text { COSO III }\end{array}$ & $\mathrm{PR}=\frac{\text { No. de proveedores re gistrados }}{\text { Total de proveedores analizados }} * 100$ & Anual & $100 \%$ & $x>66 \%$ & $\begin{array}{c}x \geq 33 \% \\
66 \%\end{array}$ & $x<32 \%$ \\
\hline
\end{tabular}

Figura 5. Mapa estratégico. 


\section{CONCLUSIONES}

La planificación estratégica en la actualidad es de importancia vital en todas las instituciones, así como para la seguridad social, la cual abarca varias perspectivas para involucrar de manera total todas sus aristas, como son, usuarios o clientes, financiero, procesos internos y conocimiento, esta planificación ayudará a las organizaciones a mantener un control y seguimiento en sus procesos, así como resultados obtenidos con la finalidad de alcanzar sus objetivos estratégicos.

En el ámbito de la seguridad social, es necesario anclar a los objetivos estratégicos la participación activa de sus afiliados, beneficiarios y empleados de todas sus unidades de negocio, con una perspectiva de ser más eficientes en sus procesos y administrar de mejor manera los recursos económicos de sus afiliados.

Es necesario también tener en cuenta la importancia de involucrar a todas las unidades provinciales del IESS, de manera igual la comunicación debe ser más asertiva para mejorar la socialización de cada uno de los objetivos y metas planteadas, así como también que estrategias deberán ser empleadas para la consecución de dichos objetivos.

\section{FINANCIAMIENTO}

No monetario.

\section{AGRADECIMIENTO}

A las unidades médicas de primer nivel de atención de salud en el área rural del IESS en la provincia del Cañar; por permitir el desarrollo de la investigación.

\section{REFERENCIAS CONSULTADAS}

Cordero-Naspud, E. I., Erazo-Álvarez, J. C., Narváez-Zurita, C. I., \& Cordero-Guzmán, D. M. (2020). Soluciones corporativas de inteligencia de negocios en las pequeñas y medianas empresas [Corporate business intelligence solutions in small and medium enterprises]. Revista Arbitrada Interdisciplinaria Koinonía, 10(5), 483-513. http://dx.doi.org/10.35381/r.k.v5i10.703 
Jorge Patricio Cañar-Rivera; Johnny Hernan Urgiles-Vicuña; Juan Diego Ochoa-Crespo Mireya Magdalena Torres-Palacios

Erazo-Álvarez, J. C., \& Narváez-Zurita, C. I. (2020). Medición y gestión del capital intelectual en la industria del cuero - calzado en Ecuador. [Measurement and management of intellectual capital in the leather industry - footwear in Ecuador]. Revista Arbitrada Interdisciplinaria Koinonía, 9(5), 437-467. http://dx.doi.org/10.35381/r.k.v5i9.662

González-Castillo, L., Hernández-Rodríguez, D., \& Palacios-Osma, J. (2017). Modelo de Simulación para evaluar las decisiones estratégicas de un comité local de AIESEC basados en el Balanced Scorecard. [Model of simulation to Evaluate Strategic Decisions in an AIESEC local committee based on the balanced scorecard]. RISTI - Revista Ibérica de Sistemas e Tecnologias de Informação, (25), 8297. https://dx.doi.org/10.17013/risti.25.82-97

Herrera-Avendaño, C, Terán, O, \& Rivera-Cruz, I. (2014). Cuadro de Mando Integral (CMI) y el Enfoque Sistémico (ES) como herramientas para la generación de valor económico del capital humano en las organizaciones. [Balanced Scorecard (BSC) and the Systemic Approach (ES) as tools for generating economic value of human capital in organizations]. Omnia, 20(2), 105-119.

Instituto Ecuatoriano de Seguridad Social (IESS, 2019). ¿Quienes somos? [About us?]. Recuperado de https://n9.cl/woo6q

kaplan, R., \& Norton, D. (2004). Mapas Estratégicos [Strategics Maps]. Barcelona: Gestión 2000. Recuperado de https://n9.cl/kh8f

Kaplan, R., \& Norton, D. (2014). El Cuadro de Mando Integral. [The Balanced Scorecard]. Grupo Planeta Spain. Recuperado de https://n9.cl/fhi2

Lazo-Torres, N. S., Erazo-Álvarez, J. C., \& Narváez-Zurita, C. I. (2019). El Balanced Scorecard como herramienta de control interno en el sector Manufacturero [The Balanced Scorecard as an internal control tool in the Manufacturing sector]. Revista Arbitrada Interdisciplinaria Koinonía, 1(4), 125-152. http://dx.doi.org/10.35381/r.k.v4i1.374

López-Zapata, E, López-Moros, G, \& Agudelo-Muñoz, S. (2019). Relación entre Estrategias Competitivas y Tipos de Aprendizaje Organizativo en Empresas Colombianas. [Relationship between Competitive Strategies and Types of Organizational Learning in Colombian Companies]. Información tecnológica, 30(5), 191-202. https://dx.doi.org/10.4067/S0718$\underline{07642019000500191}$ 
Jorge Patricio Cañar-Rivera; Johnny Hernan Urgiles-Vicuña; Juan Diego Ochoa-Crespo Mireya Magdalena Torres-Palacios

Martínez-Pedrós, D., \& Milla-Gutierrez, A. (2012). Mapas Estratégicos. [Strategic Maps]. Madrid: Ediciones Díaz de Santos. Recuperado de https://n9.cl/rdxh

Mendieta-Ortega, M. P., Erazo-Álvarez, J. C., \& Narváez-Zurita, C. I. (2020). Gestión por competencias: herramienta clave para el rendimiento laboral del talento humano del sector hospitalario [Management by competences: key tool for the labor performance of the human talent of the hospital sector]. Revista Arbitrada Interdisciplinaria Koinonía, 10(5), 287-312. http://dx.doi.org/10.35381/r.k.v5i10.696

Morales, G. (2013). ¿Qué es la Seguridad Social? [What is Social Security?]. https://n9.cl/8f9zi

Ortiz-Pérez, A, Pérez-Campaña, M, \& Velázquez-Zaldívar, R. (2014). Propuesta de cuadro de mando integral para la Universidad de Holguín. [Proposal of balanced scorecard for the University of Holguín]. Ingeniería Industrial, 35(3), 333-343.

Porter, M. (2015). Estrategia Competitiva. [Competitive strategy]. México: Grupo Editorial Patria. Recuperado de https://n9.cl/c9cx

Posso, M. (2005). La Seguridad Social que los ecuatorianos necesitamos. [The Social Security that Ecuadorians need]. Recuperado de https://n9.cl/rm1h

Rivero-Alonso, K, \& Galarza-López, J. (2017). El cuadro de mando integral como una alternativa para el seguimiento y control de la estrategia en las instituciones de educación superior. [The comprehensive chain of command as an alternative for the follow-up and control of the strategy in the higher education institutions]. Revista Cubana de Educación Superior, 36(3), 85-95.

Tarjizán, J. (2018). Fundamentos de Esrategia Empresarial. [Fundamentals of business strategy]. Santiago: Ediciones universidad católica de Chile. Recuperado de https://n9.cl/zd2w 